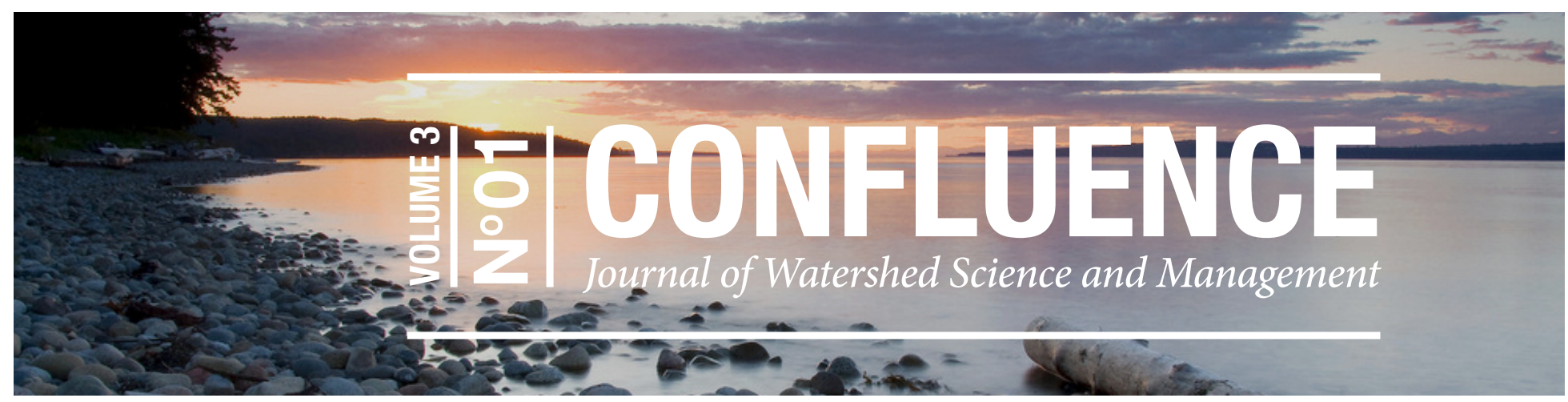

\title{
The Role of the Hydrographer in Rating Curve Development
}

\section{Stuart Hamilton, Marianne Watson, Robin Pike}

Stuart Hamilton is Senior Hydrologist at Aquatic Informatics. Email: stuart.hamilton@aquaticinformatics.com

Marianne Watson is a self-employed consulting hydrologist in New Zealand and lead writer for the NZ National Environmental Monitoring Standard for Rating Curves. Email: marianne@hydronet.co.nz

Robin Pike is a Watershed Research Hydrologist with the BC Ministry of Environment and Climate Change Strategy. Email:Robin.G.Pike@gov.bc.ca

\section{Abstract}

\section{Introduction}

Stage-discharge rating curves are used to produce most of the world's discharge data. The shape of these curves is dependent on the shape of the channel that controls flow. Changes in rating curves occur over time in response to transitory (e.g., vegetation, ice, debris) or persistent (e.g., aggradation/degradation) changes of the rated channel. Errors in rating curve development can result from the mischaracterization of the shape of the curve at a given time, or the misidentification of patterns of change over time. While data-driven methods for rating curve calibration are desirable, conventional statistical regression techniques, unfortunately, require far more data points to fully characterize the patterns of change in the curve shapes than are commonly available.

This article discusses the benefits of field observations of the stream channel in support of rating curve development. The mathematical form of the rating curve can be inferred from observations of natural channel control features that link to principles of flow. In this article, the theoretical components of the rating curve equation are discussed with emphasis on how field observations can be used to groundtruth calibrated values for the coefficient, offset, and exponent for each segment of a stage-discharge rating curve. The article also explains how conceptual models developed by the hydrographer add value to the calibration process.

A rating curve is a site- and time-specific relationship used to produce continuous records of streamflow (discharge) from water level measurements. The fundamental assumption in the stage-discharge method is that a unique discharge can be identified for any given stage. This article is applicable to gauge locations and periods of time when this assumption is true. Locations and periods of time where this assumption is false (e.g., for backwater effects or discharge hysteresis) require alternative approaches such as index velocity methods.

The basic premise of a rating curve is that water level (stage) is a reliable indicator of both the crosssectional area of water and the depth of water (which determines the velocity) where the product of area times velocity is equal to discharge (i.e., $Q=V^{*} A$ ). A rating curve is calibrated to gaugings, which are synchronized observations of water level and discharge measurements obtained during field visits (WMO 2010).

The specific shape of the rating curve depends on how rated channel features resist flow. The greater the resistance, the greater the depth of water needed to convey a given flow volume per unit of time. 
The resisting elements are collectively referred to as the "control." A channel control includes all of the features such as the shape, size, slope, roughness, alignment, constriction, and expansion of the channel, which in turn create flow resistance (Kennedy 1984).

Unfortunately, in practice, there is rarely a sufficient number of gaugings to fully characterize the shape of the rating curve (Manfreda 2018) and to correctly identify all changes in the channel that occur over time. In some instances, stream channels have complex geometry, compounding the difficulty of curve fitting from a statistical perspective. In addition, all deviations from a rating curve must be fully explained, which requires an understanding of not only the control but also the environmental and operational influences on data quality. For example, in a certain range of flow and conditions, an Acoustic Doppler Current Profiler (ADCP) may produce the most accurate measurements, whereas for a different flow range or set of conditions at the same location, a tracer-based method such as salt dilution might produce more accurate results. Therefore, awareness of the site-specific limitations of various hydrometric gauging methods is critical, as is consideration of how such errors can affect the perceived shape of the curve and extrapolation beyond field-derived gaugings.

Errors resulting from the association of gaugings to a different (given state of) control can often be problematic. Specifically, any unknown change in control conditions can lead to gaugings being wrongly associated with a given control state (i.e., a gauging made after the change should not be directly associated with any curve developed for a previous control state and vice versa). Missed changes in control state can account for substantial inaccuracies in stage-discharge relations, even outweighing measurement or calibration errors (Ibbitt and Pearson 2009). Thus, careful field observations of the control state for each gauging are necessary to understand how site- and time-specific factors might result in a rating curve change. Factors such as bedload movement, debris entrapment, bank erosion, seasonal aquatic and riparian vegetation changes, animal activities (e.g., humans, beavers), and ice effects must be considered when adjudicating gaugings for rating curve development.

The key concept in this article is that rating curve shape is a function of the hydraulic control(s) and the specific effect of the control(s) determines the parameters in the rating equation; thus, the likely range of these parameter values can be inferred in advance through field observations of channel properties. A case study of a rating curve development is used to illustrate the process for a gauge with many gaugings available for validation of a rating calibration. Strategies for dealing with more typical, complex scenarios are also discussed. This article explains how conceptual models developed by the hydrographer can add value to the rating curve calibration process.

The Rating Curve Equation
In order to explain the value of field observations, this article begins with a discussion of the theoretical basis of the conventional stage-discharge relationship. The physical form of the channel control is linked to the shape of the rating curve through the mathematical form of the stage-discharge rating equation (Equation 1) (WMO 2010; Petersen-Overleir 2005). In this equation, the parameters that define the relationship between discharge $(Q)$ and stage $(H)$ are a coefficient $\left(C_{0}\right)$, an offset $(e)$, and an exponent $(B)$ that respectively relate to the scale and resistance (to flow) of the channel, the control elevation, and the shape of the control (Equation 1).

$$
Q=C_{0}(H-e)^{B}
$$

The continuity equation (Equation 2) is the fundamental basis of the rating equation:

$$
Q=V \star A
$$

where discharge $(Q)$ is equal to the product of velocity $(V)$ times cross-sectional area $(A)$. Under open channel conditions (i.e., in the absence of ice cover), both velocity and area are almost always positively related to stage. Hence, the stage-discharge relationship is the product of a stage-area function times a stage-velocity function (Manfreda 2018). The stage-area function is dominated by the geometry of the control while the stage-velocity function is dominated by flow resistance features.

The parameters that control mean velocity of flow through the cross section can be expressed in the general form of the friction loss equation (Equation 3) (Meadows et al. 2002):

$$
V=C_{1} R^{x} S^{y}
$$


where $V$ (velocity) is the product of $C_{1}$ (a flow resistance factor), $R$ (hydraulic radius-a measure of depth, which is the ratio of the channel's cross-sectional area to its wetted perimeter), and $S$ (friction slope), and $x$ and $y$ are exponents used to represent different channel conditions (Meadows et al. 2002).

There are two common variations on the friction loss equation. The Chézy variation (aka Kutter's variation) uses exponents of 0.5 for both $x$ and $y$, and is preferred for sewer design and analysis, although it can also be applied to open channel flow (Meadows et al. 2002). For most open channel control conditions, the Manning variation (aka Gauckler-Manning or Gauckler-Manning-Strickler variation) (Equation 4), which uses exponents of 0.67 and 0.5 for $x$ and $y$, respectively, is a reliable choice:

$$
V=n^{-1} R^{0.67} S^{0.5}
$$

The Manning equation uses the inverse of a friction factor $(n)$ in place of the coefficient $\left(C_{1}\right)$ as the flow resistance factor. Manning's equation is meant for prismatic channels (i.e., rectangular, parabolic, trapezoidal) and can result in unexpected results when applied to channels where there are sudden changes in hydraulic radius (discussed further in "The Open Channel Problem" section below). The friction factor represents the sum of the effects of all resisting elements in the channel (e.g., bed material size, relative roughness, vegetation, obstructions, variations in channel width and depth, channel curvature). For practical purposes, it is not the absolute values of slope and friction factor in Equation 4 that are important, but rather the net change in flow resistance over time. Thus, the friction loss equation (Equation 4 ) can be further simplified by combining the slope and friction factor parameters into a single term $\left(C_{2}\right)$ as shown in Equation 5.

$$
V=C_{2} R^{0.67}
$$

As a simplifying rule, for alluvial channels, the coefficient $C_{2}$ (net flow resistance) can be considered to vary around unity (i.e., $C_{2}=1$ ) (Hamilton 2018) and, hence, the hydrographer can make field observations of the controlling reach at each field visit and estimate proportional change in $\mathrm{C}_{2}$ over time.

For periods of time when $C_{2}$ is unchanging, $V$ is primarily a function of hydraulic radius. However, the theoretical advantage of hydraulic radius as a predictive variable is outweighed by the practical advantage of monitoring stage and subtracting an offset value (i.e., $H-e$ ) as a continuous measure of depth, which yields a modified friction loss equation (Equation 6):

$$
V=C_{2}(H-e)^{0.67}
$$

A widely held assumption is that the offset value must be the elevation of zero flow (aka point of zero flow, cease-to-flow), as described in Rantz (1982) and Kennedy (1984). However, for channel control, the role of the offset is to model effective depth (i.e., the hydraulic radius) for any range of stage for which there is a uniformly linear stage-radius relationship. The offset value that provides the best estimate of effective depth will be different above and below any breakpoint in the stageradius relationship. Hence, for sites that have multiple controls (i.e., section, channel, overbank), there are likely multiple offsets-a unique one for each control-that cannot, logically, all be equal to the zero-flow elevation.

A simple offset to convert stage to hydraulic radius $(R)$ will work for uniform rectangular channels that are much wider than they are deep. For all other uniform shapes (e.g., as shown in Table 1), a coefficient $\left(C_{3}\right)$ is required (Equation 7):

$$
R=C_{3}(H-e)
$$

For channels with multiple hydraulic controls, the rating curve will be broken into segments, where each segment has a uniform stage-radius relationship, generally requiring a change in offset value at each transition. For channels that have a non-linear stage-radius relationship, the curve must be similarly segmented but a change in offset value is generally not required between segment transitions.

The relationship between stage and area (Equation 8) can be expressed in the same general form as Equation 6:

$$
A=C_{4}(H-e)^{b}
$$


where area $(A)$ is equal to the product of $C_{4}$ (a scaling factor generally dominated by channel width), the water level $(H)$ minus the offset $(e)$, and $b$ (a shape factor for the channel control reach). From geometry it can be deduced that the exponent for channel shape $(b)$ will be 1 if the channel is a perfect rectangle, 1.5 if the channel is parabolic in shape, and 2 if the channel is triangular (Table 1). While no natural channel will have perfect geometry, a good approximation of the value of the exponent for channel shape $(b)$ can be estimated by field observations of the channel banks within the range of stage for which a rating curve is developed (Table 1).

The product of Equations 5, 7, and 8 relates Equation 2 to Equation 1, as shown in Equation 9:

$$
Q=V \cdot A=C_{2} C_{3}^{(0.67)} \cdot C_{4}(H-e)^{(0.67+b)}=C_{0}(H-e)^{B}
$$

where discharge is the product of velocity times area, which can be expressed as functions in the same form, sharing common parameters. The product of coefficients and sum of exponents from these two equations can then be reduced to the form of Equation 1. In order to combine coefficients, the righthand side of Equation 7-which is needed to convert stage to radius for all non-rectangular geometries-has to be rearranged to move the coefficient $C_{3}$ outside of the brackets. This is achieved by raising the coefficient $C_{3}$ to the same power used in Equation 6.

Equation 9 makes explicit the link between the parameters that define the shape of the curve $\left(C_{0}, e\right.$, and $B$ ) with the physically observable features of a channel control. The coefficient $C_{0}$ is the product of the scale factor in the stage-area relationship and a flow resistance factor that includes channel slope and the friction factor. The offset $(e)$ is an adjustment that converts stage to depth of water over the control (i.e., effective depth, sometimes termed hydraulic head). The exponent $(B)$ is the sum of the shape exponent $(b)$ and the friction loss assumption exponent (e.g., 0.5 using the Chézy approach or 0.67 for the Manning approach). Given this understanding, hydrographers can better attribute deviations of gaugings from the curve to control changes, or environmental or operational factors.

Table 1. The link between various symmetric channel shapes and the rating equation is shown for various channel shapes.

\begin{tabular}{|c|c|c|c|c|}
\hline Control Shape & $\begin{array}{l}\text { Stage-Radius } \\
\mathrm{R}=\mathrm{C}_{3}(\mathrm{H}-\mathrm{e})\end{array}$ & $\begin{array}{l}\text { Stage-Area } \\
A=C_{4}(\mathrm{H}-\mathrm{e})^{\mathrm{b}}\end{array}$ & $\begin{array}{c}\text { Radius-Velocity } \\
\qquad V=C_{2} R^{x}\end{array}$ & $\begin{array}{l}\text { Stage-Discharge } \\
\qquad Q=C_{2} C_{3}^{x} C_{4}(H-e)^{(b+x)}\end{array}$ \\
\hline & $C_{3}=1$ & $b=1$ & $x=0.67$ & $\mathrm{Q}=\mathrm{C}_{0}(\mathrm{H}-\mathrm{e})^{1.67}$ \\
\hline & $C_{3}=0.67$ & $b=1.25$ & $x=0.67$ & $\mathrm{Q}=\mathrm{C}_{0}(\mathrm{H}-\mathrm{e})^{1.92}$ \\
\hline & $C_{3}=0.63$ & $b=1.5$ & $x=0.67$ & $\mathrm{Q}=\mathrm{C}_{0}(\mathrm{H}-\mathrm{e})^{2.17}$ \\
\hline & $C_{3}=0.67$ & $b=1.5$ & $x=0.67$ & $\mathrm{Q}=\mathrm{C}_{0}(\mathrm{H}-\mathrm{e})^{2.17}$ \\
\hline & $C_{3}=0.54$ & $b=1.75$ & $x=0.67$ & $\mathrm{Q}=\mathrm{C}_{0}(\mathrm{H}-\mathrm{e})^{2.42}$ \\
\hline & $C_{3}=0.5$ & $\mathrm{~b}=2.0$ & $x=0.67$ & $\mathrm{Q}=\mathrm{C}_{0}(\mathrm{H}-\mathrm{e})^{2.67}$ \\
\hline
\end{tabular}

Notes: The link between various symmetric channel shapes and the rating equation is shown for various channel shapes. The ordinate scale of these shapes is exaggerated for visualization. The radius coefficient $C_{3}$ (column 2) varies from 0.5 to 1.0 and, for any shape other than perfectly triangular or rectangular, the relationship will be slightly non-linear; however, the assumption of linearity generally holds as long as the channel width is much greater than channel depth. The shape exponent $b$ (column 3 ) varies from 1 to 2 , and the shape coefficient $\mathrm{C}_{4}$ is a function of channel width at a radius of 1 . For this example, a Manning assumption is used for the exponent $\mathrm{x}$ (column 4) and the velocity coefficient $C_{2}$ is a function of channel resistance. Column 5 shows the rating equation that can be inferred based on observations of control shape. 
Table 1 relates various symmetrical cross-sectional shapes to rating curve form. While there are an infinite number of possible channel shapes, a reasonable inference can be made about any channel shape based on this information. For example, if the right bank is a shallow trapezoid (i.e., $b=1.25$ ) and the left bank is vertical (i.e., $b=1$ ), a reasonable guess of the exponent is that it should be greater than 1 but less than 1.25. Often, it may be impossible or impractical to accurately survey the crosssectional shape of a channel control directly because: i) the control may have length (upstream from the gauge as well as downstream) and sinuosity; ii) the controlling features may run through the cross section, diagonal to the flow direction; and iii) the controlling features may be poorly defined and/or not safely accessible. The conceptual model is a substitute for rigorous survey of the hydraulic features and is a synthesis of all relevant factors relating control form and function, with the most important factors being the effective depth of water over the control and the dominant geometric shape of the channel for the active range of stage. The conceptual model can then be used to select initial parameter values, define where different segments are required, and ultimately provide boundaries in rating curve calibration. For example, a first-order estimate of the rating curve parameters $e$ and $B$ constrain the calibration problem to finding the value of $C_{0}$ with some subsequent final finetuning of $e$ and $B$ to optimize the curve fit.

There are many methods for calibrating rating curves that are dependent on hydrographer experience and intuition (e.g., Hamilton et al. 2016). While most hydrographers have an intuitive understanding of the link between control characteristics and the shape of the rating curve, the hydrographer's thought processes are best demonstrated by using the shifted log plot (WMO 2006) method of rating calibration, which is used in the following sections to trace a path between field channel observations and the final shape of the rating curve.

The Open Channel Problem
The statement made in the previous section-that hydraulic controls may require multiple segments, each with a uniform, linear, stage-radius relationship, and that these segments may require different offsets-requires further explanation. The offset will be equal to the elevation of zero flow for the lowest unique rating curve segment (i.e., if it is under section control). For channel control segments, the offset represents the water depth that is effective for generating velocity within a specific range of stage.

Figure 1 illustrates a hypothetical channel with three distinct segments that result in a non-uniform stage to hydraulic-radius relationship (Figure 1c). Note the abrupt discontinuity in hydraulic radius that results from a rapid increase in wetted perimeter (Figure $1 \mathrm{~b}$ ) at a stage of $3.5 \mathrm{~m}$ in the transition from rectangular to trapezoidal shape. This discontinuity illustrates that hydraulic radius can have unexpected results in complex channels. It is unlikely that mean velocity would be reduced by as much as shown in Figure 1f, if at all. The velocity effect is likely an artifact of hydraulic radius not being a good predictor of velocity immediately above a sudden change in width. For hydraulic radius to be a useful predictor of velocity within any range of stage, it is the stage-radius relationship contained within each prismatic segment that is relevant. Above $3.5 \mathrm{~m}$, and in spite of width being much greater than depth, the relationship between stage and hydraulic radius is slightly non-linear (Figure 1c) as the relative change in ratio of area to wetted perimeter becomes smaller as area becomes proportionally larger (Figure $1 \mathrm{~b}$ ). When the assumption of linearity between stage and radius is not valid, the curve must be broken down into smaller segments such that the assumption becomes acceptable. Equation 5 is used to calculate velocity using hydraulic radius and the simplifying assumption that $C_{2}=1.0$ for the full range of stage (Figure 1f). Discharge is calculated for the range of stage using Equation 2 (Figure 1d).

The conceptual model for this channel would have three prismatic geometries: a parabola, a deep rectangle (i.e., the red dashed line in Figure 1a), and a deep trapezoid (i.e., the green dashed line in Figure 1a). For each conceptualized shape, an offset must be estimated, where the offset is the elevation that will contain all of the water within that specific shape. This conceptualization allows for estimation of an exponent for each segment based on the geometric shape (i.e., as per Table 1). Furthermore, the problem of the non-linear stage-radius relationship above $3.5 \mathrm{~m}$ can be addressed by dividing this segment into two ranges, each with an approximately linear stage-radius relationship. 

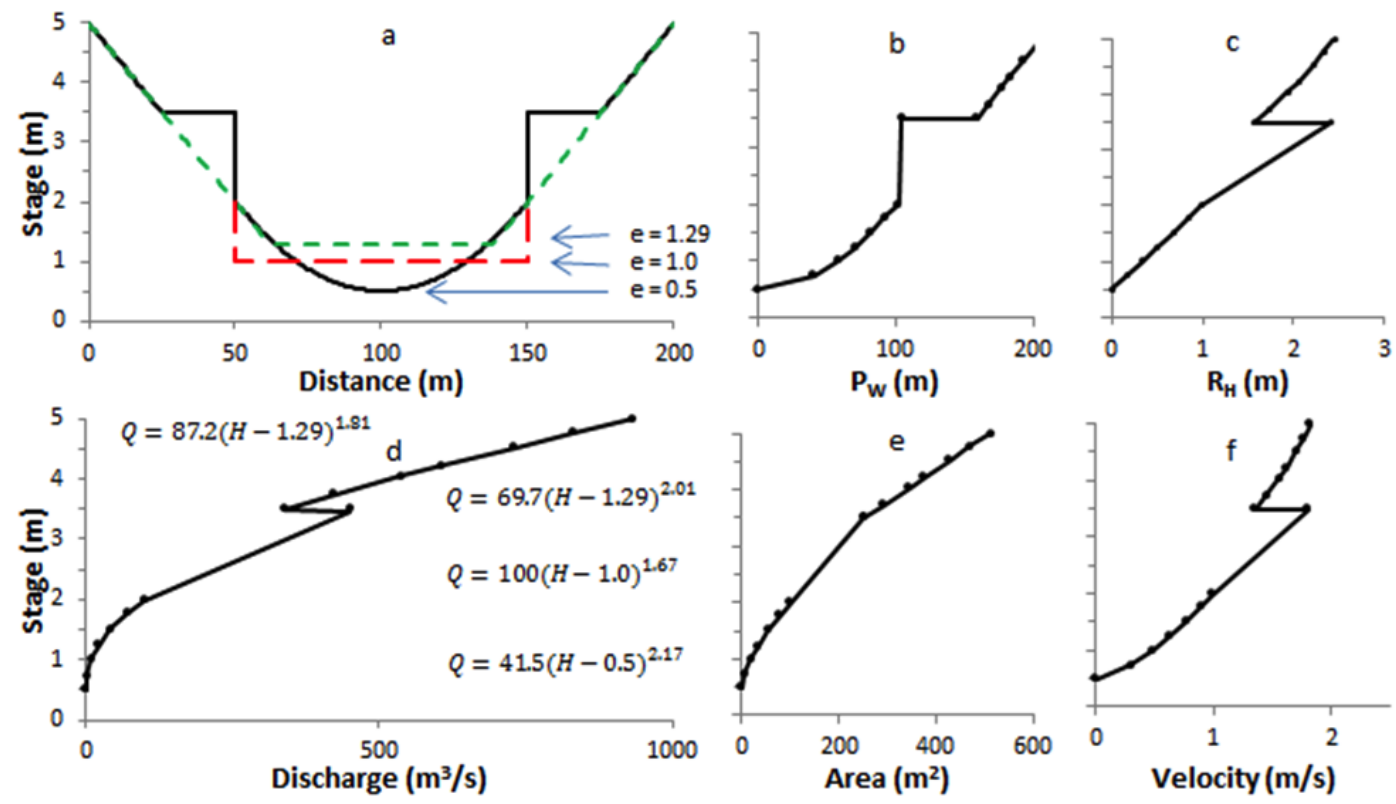

Figure 1. A hypothetical channel consisting of a parabolic segment from stage 0.5 to $2.0 \mathrm{~m}, \mathrm{a}$ rectangular segment from 2.0 to $3.5 \mathrm{~m}$, and a trapezoidal segment from 3.5 to $5.0 \mathrm{~m}$. Velocity is calculated for the range of stage using Equation 5 and the assumption that $C_{2}=1.0$. Discharge is calculated over the range of stage using Equation 2. The shifted log plot method, guided by the conceptual geometry, is used to fit a rating curve to this data. The four equations shown in $1 d$ are the product of coefficients and the sum of exponents for each curve segment where the coefficients and exponents are taken from the corresponding segments of $1 e$ and $1 f$. The offsets for these equations are taken from 1a. Note that two segments are needed in the trapezoidal region to accommodate the slight non-linearity in the stage-radius relationship shown in $1 c$.

It would take a very large number of gaugings to accurately calibrate this curve using statistical regression techniques. However, the conceptual model allows the hydrographer to develop a priori estimates of the elevations of segmentation and of the exponents and offsets needed for each segment. A benefit of field observations is that confirmation-or refutation-of the conceptual model and fine-tuning of the model parameters can be achieved using the shifted log plot method even with sparse gaugings. More importantly, the process creates a familiarity with the control features such that, over time, even relatively subtle changes in the control are noticed and documented during routine field visits.

In the next section, a conceptual model is developed for a location with a long history of gauging. A technique is proposed for evaluating the agreement between the conceptual model, the natural channel, and the underlying principles of rating curve development to illustrate how field observations and conceptual models can inform rating curve development.

Case Study of a Simple Channel Control
Data for the case study were obtained from Water Survey of Canada (WSC) for the Thompson River Near Spences Bridge (08LF051) hydrometric station in British Columbia, Canada (WSC 2018). For over 60 years, the channel has been largely stable resulting in 252 gaugings available for rating curve calibration. The gaugings span a range of stage from $0.2 \mathrm{~m}$ to $9 \mathrm{~m}$ and a cross section obtained from the cableway at the gauge covers a range of stage from $-4.7 \mathrm{~m}$ to $7.4 \mathrm{~m}$. A purpose-specific survey of the cross section of the control was not performed for this paper. However, soundings from selected discharge measurements at the cableway were rendered into the cross section presented in Figure 3. The location of the gauge and cableway (marked with a yellow pin in Figure 2) is assumed to be typical of the upstream and downstream controlling reach.

The control is an unknown length of channel, potentially extending upstream and downstream from the gauge. Hydrographers should be attentive to conditions over a sufficient length of channel to be certain that any change in the state of the channel that could alter the stage required to convey a given discharge is observed and documented, preferably with supporting photographs. 


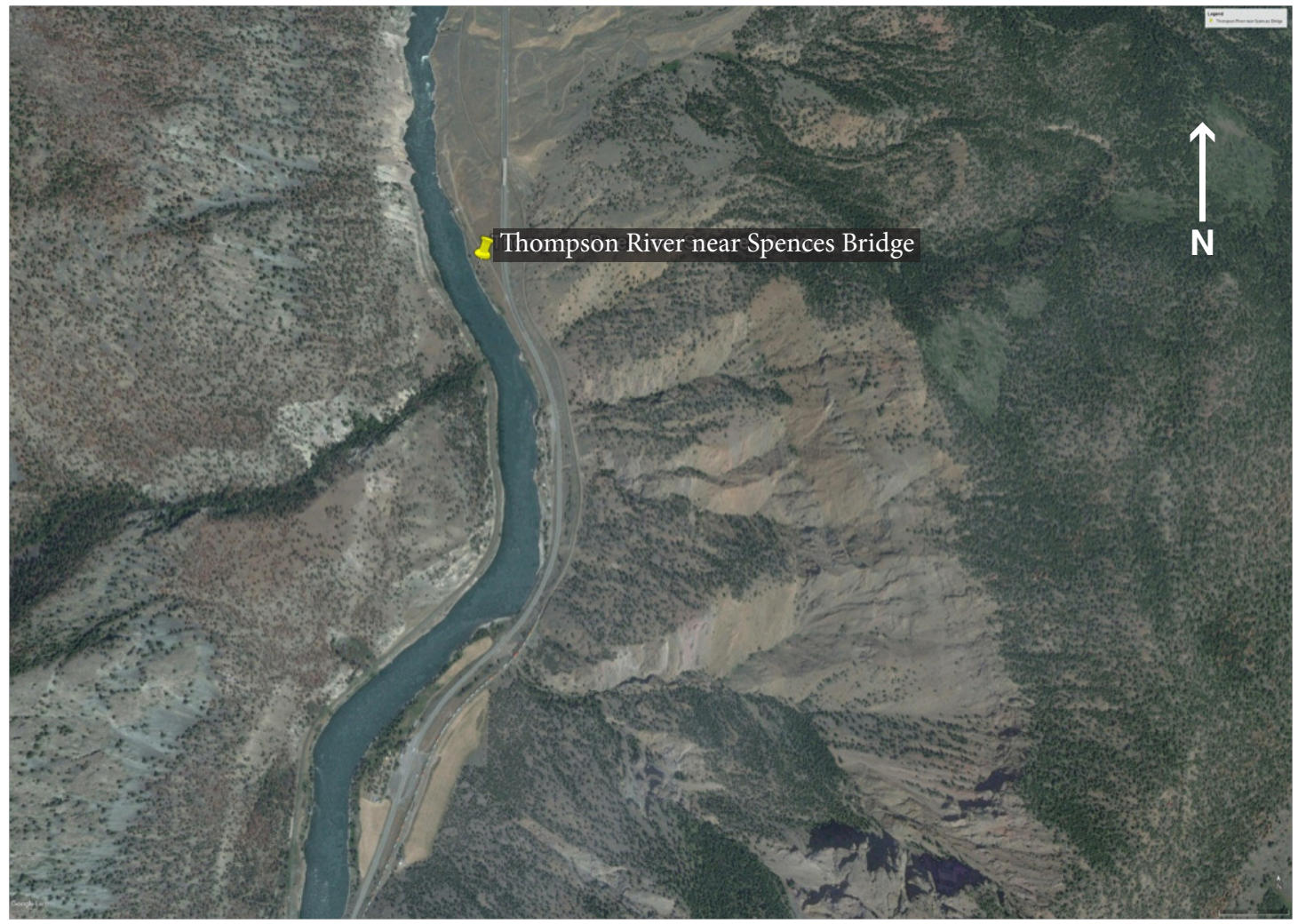

Figure 2. Thompson River Near Spences Bridge (08LF051). Direction of flow is south. There is no clearly identifiable control feature downstream of the gauge (indicated by the yellow pin) that would allow direct observation of the offset elevation (Image Source: Google Earth).

Rating curve calibration is an iterative process of conceptual model development and calibration using gaugings. In the case study, an initial working hypothesis is the channel is trapezoidal in shape and the bottom of the trapezoid is at an elevation such that the area within the trapezoid is similar to the area in the channel (Figure 3). This trapezoid has a top-width to bottom-width ratio similar to the trapezoid in row 2 of Table 1, which would predict an exponent of about 1.92. The offset is predicted from the conceptual model to be the elevation of the bottom of the trapezoid, which is $-4 \mathrm{~m}$.

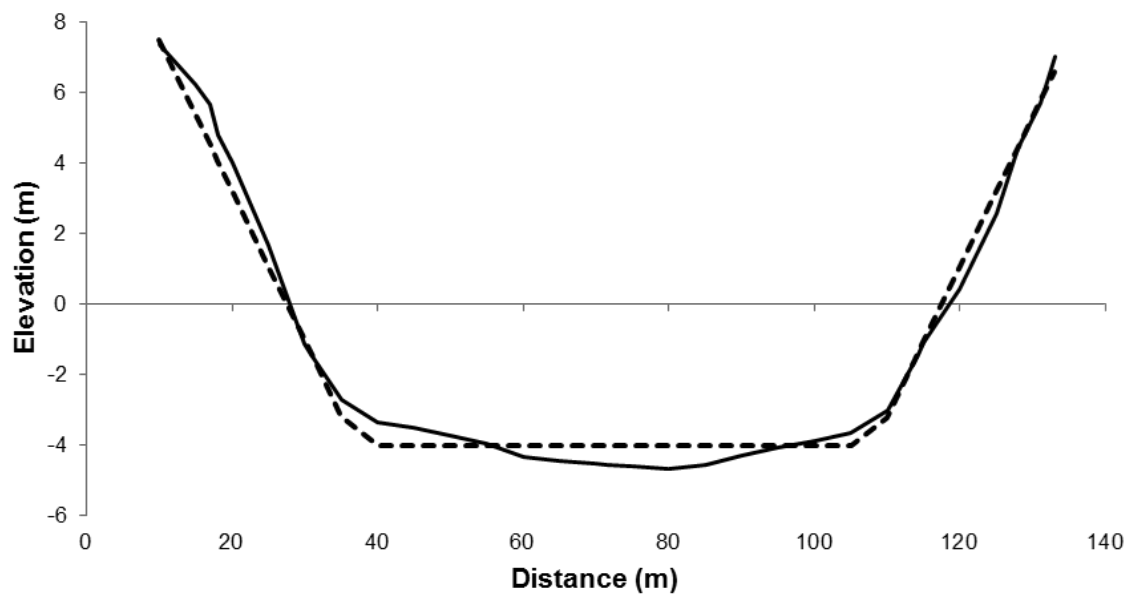

Figure 3. A conceptualization of the channel control for Thompson River Near Spences Bridge. The solid line represents the cross section at the gauge location, while the dashed line (conceptual model) demonstrates a good fit with a simple trapezoid channel shape.

The working hypothesis can be tested using the shifted log plot method with a sparse set of gaugings, specifically the first five gaugings in the period of record (Table 2). Starting from the working 
hypothesis of the offset elevation (estimated at $-4 \mathrm{~m}$ ), it is evident that the curve can readily be fit with an equation of $\mathrm{Q}=0.326(\mathrm{H}+4)^{4.13}$ (Figure 4), but has an exponent that is unrealistically high.

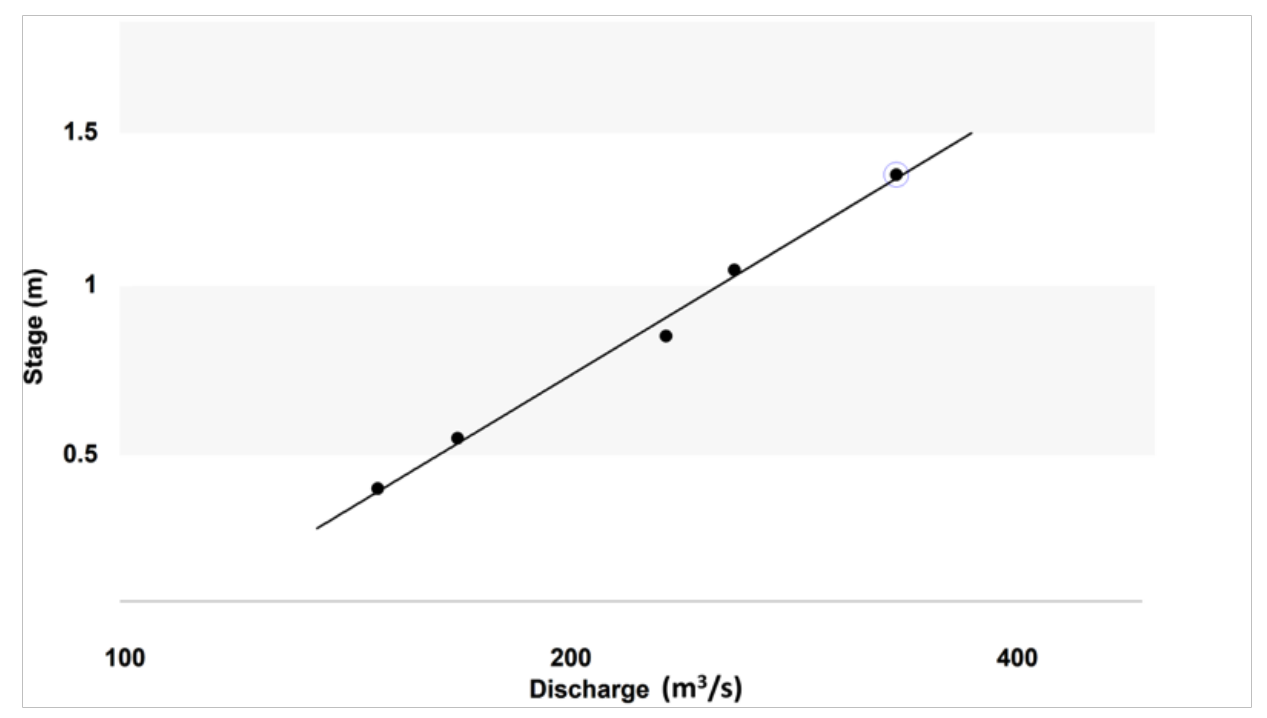

Figure 4. A shifted log plot of the gaugings with the stage axis offset by first approximation using an offset value of $-4 \mathrm{~m}$. The dashed black line shows the equation $Q=0.326(H+4)^{4.13}$. However, the exponent of the equation is larger than expected. For a deep channel that never reaches a cease-to-flow elevation, we can have more confidence in an a priori estimate of the exponent than in the offset, in which case the offset value can be adjusted to bring the rating exponent to the predicted value.

Informed by the expected value of the rating exponent, the offset elevation is then iteratively adjusted to an elevation of $-1.41 \mathrm{~m}$, at which the rating equation becomes $Q=46.97(H+1.41)^{1.916}$ (Figure 5). Remember that the coefficient is a scale factor that is dominated by channel width (but modified by other factors); hence, it should not be surprising that the curve that yields the most realistic estimate of the exponent results in a coefficient that is more closely proportional to channel width. The deviations from the two curves, as given in Table 2, show that it would be difficult to say with confidence that one curve is better than the other using statistical methods. The curves can be tested by extension to the full range of stage and using all gaugings from the period of record for validation as shown in Figure 6. As expected, prior knowledge of the shape of the rating curve improves the accuracy of curve extension into the ungauged range of stage.

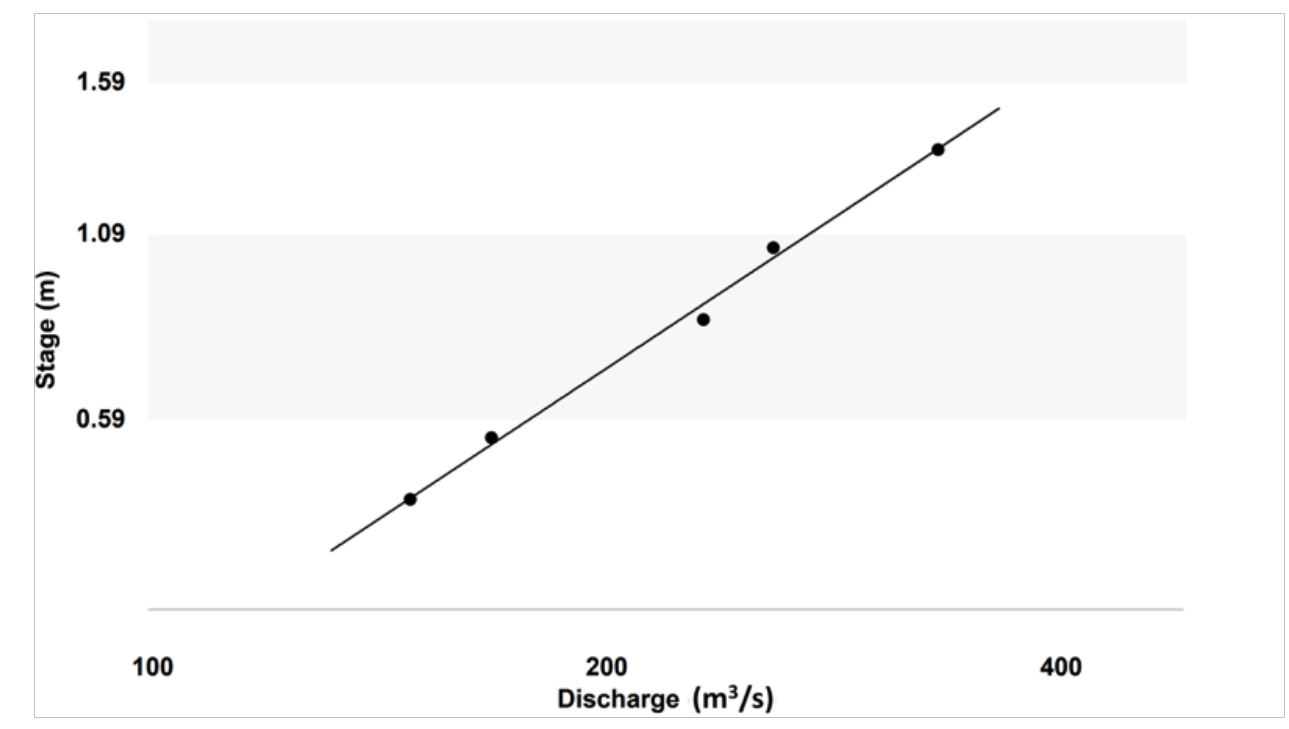

Figure 5. A shifted log plot showing a curve fit by adjustment of the offset to a value of $-1.41 \mathrm{~m}$ in order to bring the exponent to the expected value. The solid black line shows the curve with the equation: $Q=46.97(H+1.41)^{1.916}$. 
Table 2. Gaugings used to calibrate the rating curve.

\begin{tabular}{|l|c|c|c|c|}
\hline Date & Stage $(\mathrm{m})$ & Discharge $\left(\mathrm{m}^{3} / \mathrm{s}\right)$ & Error $(\%)$ & Shift $(\mathrm{m})$ \\
\hline $1951-09-28$ & 1.359 & 331 & $0.31(-0.98)$ & $0.004(-0.013)$ \\
\hline $1951-11-08$ & 1.052 & 258 & $-2.21(-1.67)$ & $-0.029(-0.021)$ \\
\hline $1951-11-23$ & 0.847 & 232 & $3.77(4.81)$ & $0.044(0.055)$ \\
\hline $1952-01-09$ & 0.549 & 168 & $-1.41(-1.36)$ & $-0.015(-0.015)$ \\
\hline $1952-03-25$ & 0.408 & 148 & $0.42(-0.83)$ & $0.004(-0.009)$ \\
\hline
\end{tabular}

Note: Deviations in discharge $(\%)$ and stage $(\mathrm{m})$ relative to the curve $\mathrm{Q}=46.97(\mathrm{H}+1.41)^{1.916}$ (Figure 5) are given in columns 4 and 5. Deviations relative to the curve $\mathrm{Q}=0.326(\mathrm{H}+4)^{4.13}$ (Figure 4 ) are shown in parentheses in columns 4 and 5. The deviation statistics are Error, which is the percentage of deviation of the measured discharge from the curve, and Shift, which is the length of deviation of the measured stage from the curve.

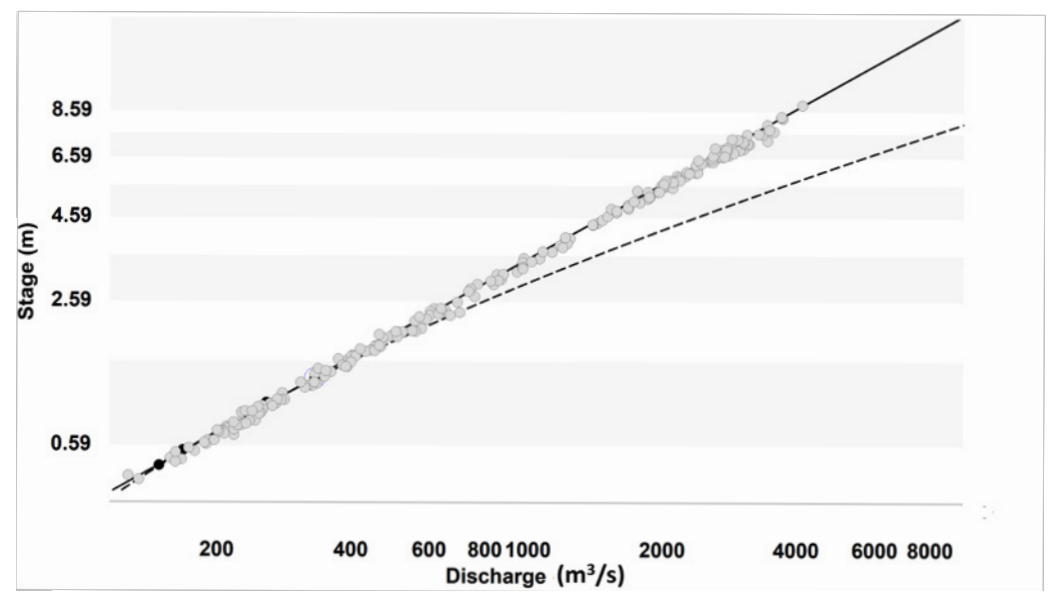

Figure 6. The solid black line is the rating with an equation of $Q=46.97(H+1.41)^{1.916}$ (from Figure 5) after it is extrapolated to the full range of stage and tested against the full set of gaugings (grey dots). The dashed black line is the curve with an equation of $Q=0.326(H+4)^{4.13}$ (from Figure 4), extraolated to the full range of stage. The calibration gaugings from Table 2 are shown as black dots, which are mostly hidden by validation gaugings. Note that with the offset value added to the ordinate axis scale, the gaugings plot as a straight line. By using this method, errors in the offset value can be readily identified because if the offset is too low the gaugings will plot in a concave up pattern, or if too high the gaugings will plot in a concave down pattern.

The revised conceptual model can further be investigated by field observations. In this case, the conceptualization of the channel control requires an offset of $-1.41 \mathrm{~m}$ to adequately fit the data for the gauged range of stage. It is important to remember that this offset for channel control is not the ceaseto-flow elevation. The lowest gauged discharge is $126 \mathrm{~m}^{3} / \mathrm{s}$ and it is entirely possible that if the flow were to actually drop to zero that a section control would become apparent, with a much different offset value. A section control has not been observed at low water levels, though it is possible that one could be revealed by bathymetric surveys of the channel reach. Each iteration in the conceptualization and calibration of any rating curve results in refinement of the fundamental assumptions about control form and function, which can be examined by field observations. Hence, the hydrographer is not solely dependent on gaugings to confirm that the curve is a reasonable approximation of the "true" curve.

Furthermore, every measurement that does not plot within uncertainty bounds must be investigated because those measurements may indicate measurement error, errors in model structure, or errors in assumptions made about control stability. The model calibration process is only complete when all deviations are fully explained.

Gauge data can be reconciled with conceived area and velocity through the controlling reach using the principle of flow continuity. The first step is to calculate an effective area (i.e., the cross-sectional area over the control $-A_{f}$ ) by subtracting the cross-sectional area that is ineffective for flow below the stage offset $\left(A_{e}\right)$ using Equation 10.

$$
A_{f}=A-A_{e}
$$


The second step is to calculate an effective velocity (i.e., the mean velocity for the effective area over the control- $V_{f}$ ) by dividing measured discharge by the computed effective area using Equation 11.

$$
V_{f}=\left(\frac{Q}{A_{f}}\right)
$$

The results of these transformations are shown in Figure 7. The conceptual model can be reconciled with the measured data using this technique as long as the assumption that the geometry of the control reach is similar to the geometry of the surveyed cross section for the measured range of stage is true. In this case, the assumption may be true because the product of coefficients (i.e., $0.58 \times 82.07=$ 47.6) and sum of exponents (i.e., $0.825+1.097=1.92$ ) for Equations 6 and 8 are in relatively close agreement with the coefficient and exponent (46.97 and 1.916, respectively) of the calibrated rating curve (Figure 5). It should be noted that this surveyed cross section is somewhat steeper-sided than predicted (i.e., 1.097 rather than 1.25 [Table 1]), and the Manning friction loss assumption (Equation 5) underestimates the velocity exponent (i.e., 0.825 rather than 0.67 [Table 1]). These compensating errors may be due to either the cross section not being representative of the controlling reach or the Manning assumption not being valid for this channel.
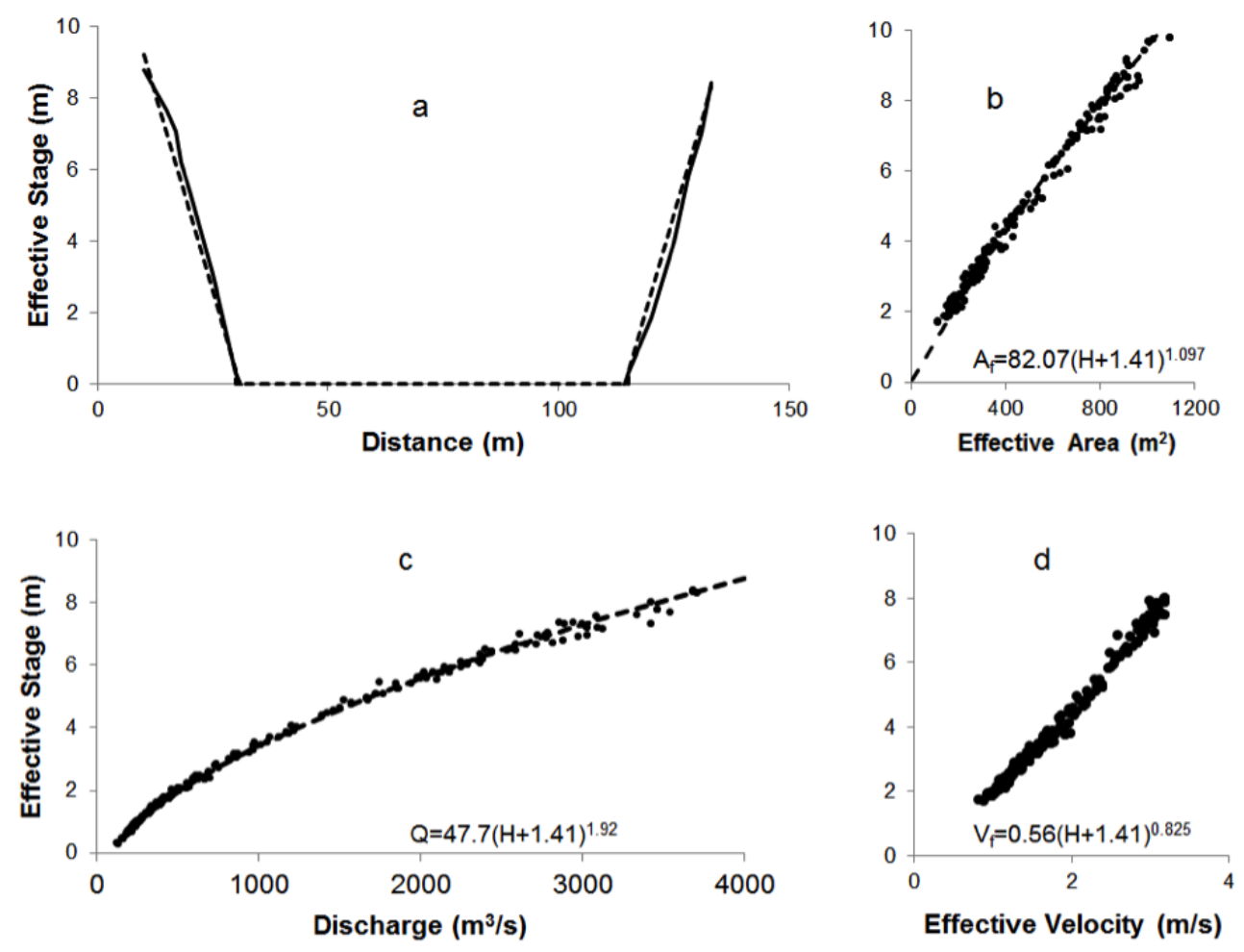

Figure 7. A plot comparing the conceptual model to measured data by plotting effective stage against effective area calculated with Equation 10 (7b) and effective velocity calculated with Equation 11 (7d). The product of coefficients and sum of exponents of these relationships agree reasonably closely with the calibrated coefficient and exponent of the rating curve (Figure 5) as predicted by Equation 9.

Further discussion about curve reliability is not solely dependent on the statistical goodness of fit. This rating process exposes the assumptions implicit in the development of the rating curve and thoughtful consideration of these assumptions will not only lead to further improvements in the curve but also to improvements in the hydrographer's ongoing observations of the controlling reach.

The shifted log plot (WMO 2006) is generally sufficient to test that the selected offset represents the elevation of zero effective depth for any segment of the rating curve. Iteration of the offset value guided by the shape of the gaugings in the shifted log plot allows for quick discovery of a robust offset value to use for the rating curve segment. The technique of using Equations 10 and 11 -which provides further justification that the selected offset relates the curve equation to underlying principles as shown in Equation 9-is presented primarily as a learning aid. This article recommends that hydrographers use this technique to deconstruct their ratings in the context of measured cross sections to investigate underlying relationships. 
Applying Key Concepts to Less than Ideal Locations
Every effort should be made to locate gauging stations at sites where the hydraulic control is as ideal as possible. However, it is often the case that even the best available site is far from ideal. In fact, the case study for the Thompson River Near Spences Bridge hydrometric station is unusual, considering it has a high number of gaugings over the full range of stage, the curve is uniform, and there is only one hydraulic control in effect at all stages for the entire period of record. This combination of conditions is almost never the case for most hydrometric locations. More typically, one or more of the following conditions are encountered: i) gaugings are sparse; ii) systematic errors exist; iii) there are multiple controls; iv) transient environmental factors (e.g., vegetation, ice, debris) alter the control; v) the channel is aggrading (i.e., gaining sediments) or degrading (i.e., losing sediments); and/or vi) the streambed is mobile (e.g., sand-bed streams). The following section describes how each of these conditions can be mitigated by the hydrographer using field observations and the method developed in this article.

\section{Sparse gaugings}

When gaugings are sparse, a robust concept of the "true" shape of the rating curve is important. Furthermore, as a curve increases in complexity (i.e., the number of hydraulic controls), the number of gaugings required increases proportionally. With very few gaugings, measurement errors are unlikely to cancel out, which can lead to a "best-fit" curve that embodies measurement error and, hence, is unrealistic. Further, interpolation between gaugings and extrapolation beyond gaugings can be misguided due to a lack of data. If the hydrographer understands how a "realistic" curve should look based on consideration of the controlling features, then a good rating can be developed from very few gaugings per hydraulic control.

\section{Systematic errors}

Systematic errors (e.g., as a result of an unstable reference gauge) will result in a "best-fit" rating curve that may seem reasonable on first inspection but has unrealistic values for the offset, coefficient, and/or exponent values. Any rating curve segment that has an offset value that does not closely conform to field observations of the height of the control, or has an exponent value that is either less than 1.5 or greater than 3 , is likely an artifact of some form of systematic error. A good understanding of what the rating curve "should" be as a comparison to the "best-fit" curve is often the first clue in the discovery of otherwise hidden systematic errors.

\section{Multiple hydraulic controls}

Whenever hydrographers are overly focused on goodness-of-fit criteria, there is a tendency to improve statistical fit by introducing complexity to the rating curves. However, a more complex curve may actually underperform for discharge prediction (Sörengård \& Di Baldassarre 2017). Judgement about rating complexity is best informed by field observations of the controlling reach. This assessment includes decisions about how many segments are needed, the specific elevations of transition from one segment to the next, and what factors influence curve shape for each segment. Often the best rating curve is one that is as simple as possible.

\section{Transient environmental conditions}

A large number of environmental conditions can introduce transient changes to flow resistance, channel geometry, or both. Such changes, if unnoticed, can result in very substantial rating curve errors. Conditions that primarily affect either bed elevation or wetted perimeter generally affect the offset parameter. Conditions that primarily affect channel slope, channel width, or flow resistance generally alter the coefficient parameter. Conditions that alter the side slope of banks generally affect the exponent parameter. Using this knowledge, the hydrographer can skillfully shape a new curve, or a shift correction, using as few as one gauging to make the adjustment.

\section{The channel is aggrading or degrading}

Channel controls in high-energy streams are problematic because virtually every storm event that is big enough to get a high-stage gauging-needed to define the shape of the curve-is also big enough to alter the channel control, making previous gaugings irrelevant to the existing curve under development. For many coastal mountain streams, rating curves are only valid for one storm cycle, which may only be long enough to get zero, one, or two gaugings, generally at less than peak water levels.

As poorly suited as stage is to predict discharge under these circumstances, the stage-discharge rating curve is usually the only viable choice for continuous monitoring of streamflow. Highly 
skilled rating curve analysis is needed when gaugings for a given control are sparse because the channel is constantly changing. One approach that has proven to be fairly reliable for streams like this is to create a type curve as described by McKerchar and Henderson (1987), and vertically offset the curve as needed for each unique period of quasi-stable conditions.

As illustrated in Figure 8, the type-curve approach is based on the general assumption that neither the channel shape nor net-flow resistance change over time, leaving change in bed elevation-due to sediment transport-as the dominant cause of rating curve change. This assumption means that the exponent and coefficient are deemed to be stable over time. This set of assumptions is simplistic; however, given the sparse gauging frequency, a more complex set of assumptions would be difficult to justify. A realistic field estimate can help calibrate the exponent and coefficient of the type curve for periods that are believed to be quasi-stable (e.g., the blue curve in Figure 8). Once the type curve is established, vertical offsetting can be achieved by either adjusting the offset value (while keeping the coefficient and exponent the same) as shown in Figure 8 or by applying a shift correction. Either a single point shift correction or an adjustment of the offset value by the same amount will result in a curve that is vertically displaced by that amount in a plot of the curve with stage on the ordinate axis.

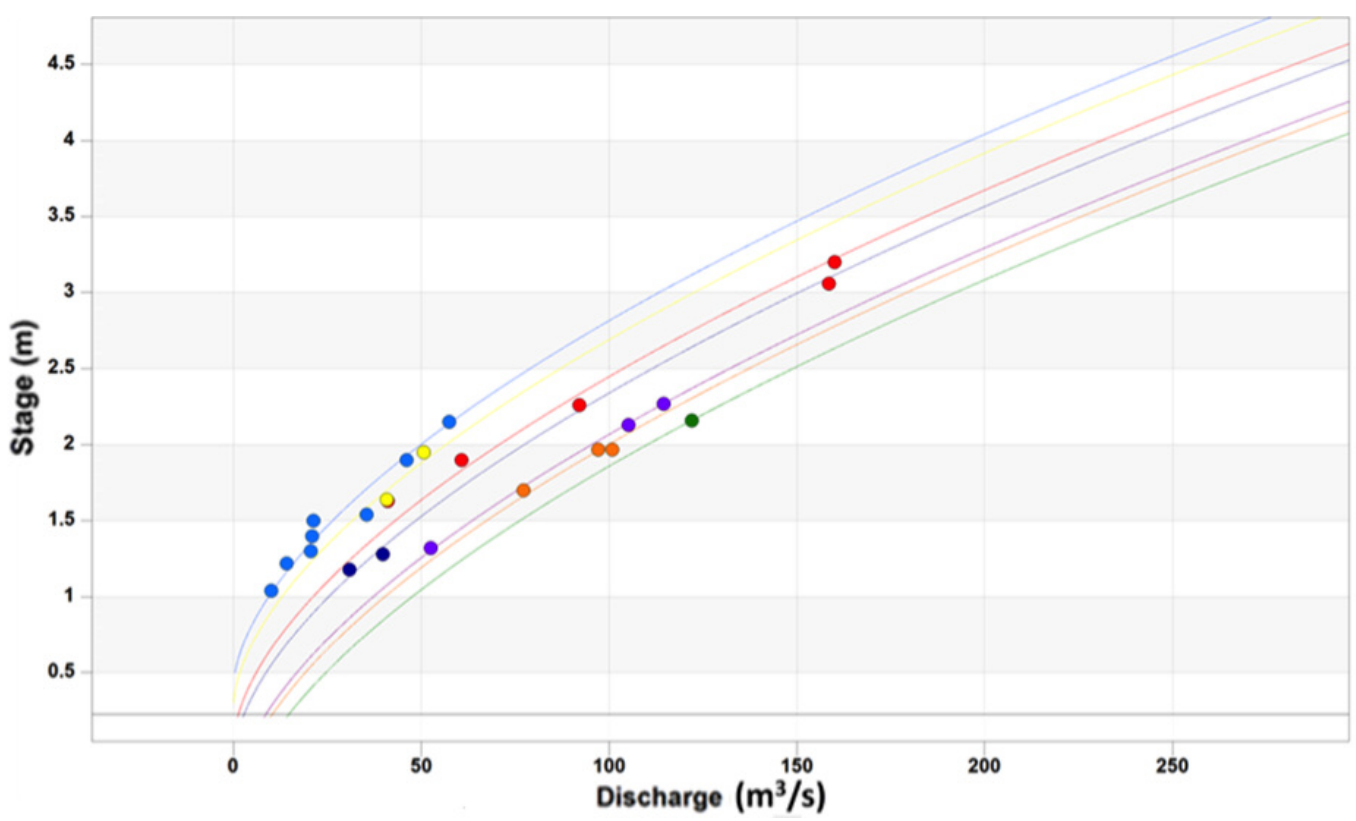

Figure 8. Example of the use of type curves. Ratings are organized sequentially in time from red, orange, yellow, green, blue, indigo, and violet over a six-year period. The ratings all have the same exponent and coefficient and differ only in their offset value, assuming that the dominant change in control is due to changes in the streambed elevation as the stream aggrades and degrades over time. Note that the green curve is calibrated to a single gauging.

\section{Mobile streambed}

Mobile streambeds are distinguished, for the context of this discussion, from aggrading or degrading channels because the former are often low-energy, supply-driven systems, whereas the latter are often supply-variable, energy-driven systems. Streams that run through extensive deposits of sand are essentially supply-unlimited and it generally doesn't take a great deal of energy to mobilize a sand-bed stream. According to the general principles of scour and fill (Colby 1964), the net effect should approach zero over a long enough period of time if the upstream source of sand is unlimited.

If the general assumptions implicit in the type-curve approach can be deemed to be valid for use in a mobile streambed then the only thing that distinguishes the analysis of rating curves for mobile streambeds from the previous discussion for aggrading or degrading streams is the process of calibration of the type curve. If, and only if, there is a sufficiently large number of gaugings then the type curve can be calibrated based on the assumption that the net effect of bed movement is zero. Hence, errors in the curve due to uncertainty of bed elevation can collectively be treated as random errors that will average out. 
Application of Field Observations to Rating Curve Development
A conceptual model informed by many field visits and inclusive of all of the elements described below can make rating curve analysis a relatively straightforward process, even for the most difficult gauging locations. The conceptual model provides a basis for using gaugings as a test of a viable working hypothesis and for refinement of the model, rather than using them for statistical calibration of the rating. The key point is that field measurements provide a validation of fundamental assumptions prior to minor refinements by calibration; something that pure statistical regression cannot equal. The conceptual model is, ideally, developed as a result of observing flow dynamics through the controlling reach at all stages and seasons and before, during, and after control-altering events. Field observations should focus on addressing the following eight topics.

\section{Uncertainty}

The "true" uncertainty of stage and discharge must be estimated for all measurement methods and environmental conditions. Sometimes the largest limiting uncertainty is stage, in which case deviations in the vertical dimension are concerning, whereas for discharge measurement, error deviations in the horizontal dimension determine whether a gauging is within rated tolerance.

\section{Sensitivity}

Sensitivity of the rating curve is the ratio of change in stage for a given change in discharge (NEMS 2016). If discharge monitoring is required for a control with low sensitivity (e.g., a wide shallow riffle), some channel modification may be required (e.g., addition of natural boulders or construction of an artificial control structure) in order to get a more sensitive rating curve. However, in some channels at very small flows (e.g., wide channels conveying less than $20 \mathrm{l} / \mathrm{s}$ ), rated discharge uncertainty is more a function of stage measurement uncertainty (Freestone 1983). In such a case, stage measurement resolution must also be improved to avoid compromising the accuracy of the rated discharge.

\section{Stability}

Identifying periods of time when the control is stable is one of the greatest challenges in rating curve development. Field observations should focus on the channel beds and banks for any change in control elevation, shape, and factors affecting flow resistance. Observations of what has changed in a control should be supported by inference about how the process occurred so that the timing of the change event can be properly represented in the rating model. For example, it is desirable to maintain the control in a pristine state, which may require the removal of weeds or debris. Whereas the accumulation of weeds may be gradual over weeks or months, the removal by cleaning of the control is abrupt. When such maintenance is performed, gaugings are needed pre- and post-cleaning to correctly identify the rating curve in both states. One field method of monitoring the onset and/or rate of change in channel stability is to take periodic photographs of the control reach using a control point, either manually on every site visit or automatically on daily or finer sampling intervals using a trail camera. Date- and time-stamped photographs can become important metadata in the rating curve delineation process over time.

\section{Complexity}

A key theme of this article is that rating curves should be supported by conceptual models that relate the physical control features to the rating curve segments. This can be specified from field observations of the elevations at which a change is expected and the corresponding expected change in curve form (i.e., increase or decrease of the coefficient, offset, and exponent).

\section{Extrapolation and Interpolation}

It is often necessary to extend a curve beyond the range for which gaugings are available to validate the curve shape. Field observations can help to identify the limits of rating curve validity. The extrapolation of a curve beyond gaugings should not extend beyond the range explained by a conceptual model (i.e., the range of the curve should not extend beyond the hydraulic control(s) that define that curve). Interpolation can also be a problem for curves with sparse gaugings. Consider the curves shown in Figures 4 and 5 where there are too few gaugings to have empirical confidence that one curve predicts the shape of the rating better than the other. It is only by consideration of the conceptual model that the curve with the most realistic shape becomes evident.

\section{Offset}

For the lowest discharge segment of the rating curve, the offset can be approximated by observing 
and/or estimating the depth of flow through the control reach. For each segment covering higher discharges, an estimate can be made from observations about whether the effective depth should increase or decrease relative to the first offset. For example, observation of a channel that looks like Figure 1 would yield the conclusion that the segment 1 offset is about $0.5 \mathrm{~m}$, the offset for segment 2 is somewhat higher, and the offset for segment 3 is somewhat higher still. The field estimate of the offset does not need to be precise to be useful. An educated guess that has decimeter-scale accuracy is usually enough to ensure that the offset determined by calibration is realistic (i.e., correct for the right reasons).

\section{Exponent}

As presented in this article, the exponent is the sum of the geometry and the friction loss components. The Chézy assumption is valid for critical velocity, whereas the Manning assumption is valid for most open-channel flow conditions. Field observations of segment-specific channel shape can provide important information for selecting the initial exponent value. Better yet is a survey of the hydraulic control cross section from which the area exponent can be explicitly determined. All assumptions need to be evaluated based on field observations of actual conditions. For example, streams with very high roughness may result in friction loss exponents greater than 0.67 .

\section{Coefficient}

The coefficient is a scale and channel resistance factor that is not directly observable by inspection of the control. However, for a rectangular channel, the coefficient value will be dominated by channel width, which can be used as a first estimate. For all other shapes, the coefficient is dominated by the channel width at an effective depth of 1 (i.e., area is equal to width multiplied by depth and a depth of 1 neutralizes the exponent of the depth-area relationship), so the coefficient can't be estimated independently of the offset estimate. In every case, the coefficient will vary in response to changes in the friction factor and/or channel slope. It will decrease with any increase in channel roughness or decrease in channel slope, and vice versa. Field observations of the factors controlling friction loss are very useful for predicting changes in a rating curve over time through coefficient changes. A change in the coefficient rotates the curve clockwise (increase) or counter-clockwise (decrease) in an arithmetic plot and shifts the curve right (increase) or left (decrease) in a logarithmic plot.

\section{Conclusion}

The formation of a conceptual model from field observations of the control features, including at what stages they are in effect and how those features change over time, is a key step in rating curve development. This knowledge ensures that the alignment of gaugings on a shifted log plot represents a uniform curve segment that is logical and, therefore, is not the result of chance or unexamined assumptions. In developing the rating curve, a sufficient number of gaugings must be collected under similar control conditions to test the model. Field observations for each gauging provide insight into when and how field conditions have likely changed (and, hence, when and how to alter the rating curve). Field observations also support the definition of offset elevations for each curve segment; while for most channel controls, there is no precise way to obtain this offset elevation by direct measurement, simple field observations of the height of the controlling reach are often sufficient to estimate approximations of the offset that are useful to ensure the calibrated value is realistic. Above all, testing the conceptual model of the rated reach with gaugings creates a continuous learning process, iteratively improving the understanding of the control, which results in a better attribution of deviations from the rating curve to measurement error, assumption error, control state change, or rating curve error.

This article emphasizes the importance of documenting hydrographer field experience systematically through metadata in the form of comments and photographs attached to every field visit. All of this metadata should be easily accessible for the purpose of sorting and filtering gaugings for developing rating curve relations. Similarly, gauging details should be easily accessible to validate that the method, technology, and technique of measurement are well suited for the conditions of measurement. Confidence in measurement results is especially important for gaugings that have high influence on the acceptance or rejection of a working hypothesis. The ultimate role of the hydrographer and their respective field observations is to link first-hand, site-specific field experience with a robust mathematical basis for the rating curve development. 
Colby, B.R. 1964. Scour and fill in sand-bed streams. USGS Professional Paper 462-D. Washinton, DC: U.S. Department of the Interior. https://pubs.usgs.gov/pp/0462d/report.pdf (Accessed January 21, 2019).

Freestone, H.J. 1983. The sensitivity of flow measurement to stage errors for New Zealand catchments (Note). Journal of Hydrology 22(2). http://www.hydrologynz.co.nz/downloads /JoHNZ_1983_v22_2_Freestone.pdf (Accessed January 21, 2019).

Hamilton, S. 2018. A question of unity - calculating the velocity coefficient. Aquatic Informatics blog post. (Updated November 13, 2018.) https://aquaticinformatics.com/blog/question-unitycalculating-velocity-coefficient/ (Accessed January 21, 2019).

Hamilton, S., R. Maynard, \& T. Kenney. 2016. Comparative investigation of Canadian, US, and Australian stage-discharge rating curve development. Australian Hydrographers Association 2016 Conference, Oct. 24-27, 2016, pp. 1-24. Canberra, AU. www.researchgate.net/profile /Stuart_Hamilton4/publication/309652693_Comparative_Investigation_of_Canadian_US _and_Australian_StageDischarge_Rating_Curve_Development/links/581bb0e808aeccc08 aea8384.pdf (Accessed January 21, 2019).

Ibbitt, R.P., \& C.P. Pearson. 2009. Gauging frequency and detection of rating changes. Hydrological Science Journal, 32(1):85-103. doi: https://doi.org/10.1080/02626668709491164

Kennedy, E.J. 1984. Discharge ratings at gaging stations. In Book 3: Applications of hydraulics. USGS Techniques of Water-Resources Investigations 03-A10. Washington, DC: U.S. Geological Survey. https://pubs.usgs.gov/twri/twri3-a10/pdf/TWRI_3-A10.pdf (Accessed January 21, 2019).

Manfreda, S. 2018. On the derivation of flow rating curves in data-scarce environments. Journal of Hydrology, 562:151-154. https://doi.org/10.1016/j.jhydrol.2018.04.058

McKerchar, A.I., \& R.D. Henderson. 1987. Drawing and checking stage/discharge rating curves. Publication No. 11. Hydrology Centre, Christchurch. NZ. 29p.

Meadows, M. E., T.M. Walski, T.E. Barnard, \& S.R. Durrans. 2002. Computer applications in hydraulic engineering. Waterbury, CN.: Haestad Press. www.dynatech.nl/Basic\%20Hydraulic \%20Principles.pdf (Accessed January 21, 2019).

National Environmental Monitoring Standards (NEMS). 2016. Rating curves: Construction of stagedischarge and velocity-index ratings: Version 1. NZ: NEMS. www.lawa.org.nz/media/2632366 /NEMS-Ratings-Feb-2016.pdf (Accessed January 21, 2019).

Petersen-Overleir, A. 2005. A hydraulics perspective on the power-law stage-discharge rating curve. Norges vassdrags- og energidirektorat Rep. 5-05. http://publikasjoner.nve.no/report/2005 /report2005_05.pdf (Accessed January 21, 2019).

Rantz, S.E. 1982. Measurement and computation of streamflow: Volume 2. Computation of discharge. Water Supply Paper 2175. Washington, DC: U.S. Geological Survey. https://pubs.usgs .gov/wsp/wsp2175/pdf/WSP2175_vol2a.pdf (Accessed January 21, 2019).

Sörengård, M., \& G. Di Baldassarre. 2017. Simple vs complex rating curves: Accounting for measurement uncertainty, slope ratio and sample size. Hydrological Sciences Journal, 62(13):2072-2082. doi :10.1080/02626667.2017.1367397

Water Survey of Canada (WSC). 2018. WSC field visit data request. (July 10, 2015.) https:// wateroffice.ec.gc.ca/ (Accessed January 21, 2019).

World Meteorological Organization (WMO). 2006. Technical regulations - volume III - hydrology, No. 49, 2006 Edition. Geneva, CH: Secretariat of the World Meteorological Organization. https://library.wmo.int/pmb_ged/wmo_49-v3-2006_en.pdf_(Accessed January 21, 2019).

World Meteorological Organization (WMO). 2010. Manual on stream gauging - volume II - computation of discharge. No. 1044. Geneva, CH: Secretariat of the World Meteorological Organization. https://library.wmo.int/pmb_ged/wmo_1044-v2_en.pdf (Accessed January 21, 2019). 